\title{
Caracterización de la población usuaria que ingresa al programa interrupción legal del embarazo en un hospital del sector salud del DF
}

\section{A D.F. Health Sector Hospital Legal Pregnancy Interruption Program User Population}

Mtra Martha Lilia Bernal Becerril; Hernández Ruiz Yoali Yeyetlzi•• Mercader Arroyo Ma. Fernanda,.• Guerrero Labastida Alma Teresa.• Mtra. Gandhy Ponce Gómez •

\section{Resumen}

Introducción: El embarazo no deseado, lleva a la mujer a practicarse un aborto. De acuerdo a International Projects Assistance Services (IPAS) en México, el aborto y sus complicaciones son la $5^{\text {a }}$ causa de muerte materna. En el D.F. la despenalización de la interrupción del embarazo antes de las 12 semanas de gestación es una ley, lo que ha significado derecho a elegir voluntaria y libremente sobre la maternidad y por lo tanto a decidir la interrupción de un embarazo. Objetivo: Caracterizar a la población que acude al programa de Interrupción Legal del Embarazo (ILE) en un hospital del sector salud del D.F. Metodologia: Estudio cuantitativo, descriptivo y transversal, encuestando a 61 mujeres, que deseaban ingresar al programa ILE y aceptaron participar en el estudio. Se realizó y aplicó un instrumento de valoración con 27 ítems. Resultados: Mujeres asistentes al ILE de entre 16 y 42 años; el $46 \%$ se dedican al hogar; $50 \%$ solteras y el resto en unión libre o casadas; el 23\% reportó no emplear ningún método para control de la natalidad. Principales razones para acudir al programa ILE: problemas económicos $(36 \%)$, embarazo no deseado $(20 \%)$, paridad satisfecha $(20 \%)$. Principal sentimiento que despierta el ingreso al programa ILE: tranquilidad (82\%). El 100\% de las mujeres sometidas a ILE aseguran haber sido tratadas con ética y dignidad. Discusión: Se concuerda con Gutmacher que la legalización de la interrupción del embarazo coincide con el incremento en solicitudes de la mujer a este procedimiento. Respecto a las razones tales como embarazo no deseado y los proble-

- Facultad de Enfermería de la Universidad Autónoma de Tamaulipas; Cd. Victoria, Tamaulipas. Correspondencia: Facultad de Enfermeria Cd. Victoria, Tamaulipas. Universidad Autónoma de Tamaulipas.

Tel.-Fax (834) 3121832•Email. obanda@uat.edu.mx obanda@uat.edu.mx

RECIBIDO: 28 DE AGOSTO DE 2009.

ENVIADO A CORRECIONES: 3 DE SEPTIEMBRE DE 2009.

ACEPTADO: 4 DE DICIEMBRE DE 2010 
mas económicos, ello corresponde con los resultados de la encuesta IPSOS- Bimsa para Population Council en la población Mexicana. Conclusiones. Debe ser creciente la calidad de atención a la salud reproductiva, se debe priorizar en la prevención de embarazos no deseados, orientando y educando a la población y ofreciendo métodos de planificación a hombres y mujeres con vida sexual activa.

Key Words:

Legal Pregnancy interruption program

\section{INTRODUCCIÓN}

El embarazo no deseado y el aborto realizado en condiciones inseguras representan problemas de gran complejidad dentro del campo de la salud reproductiva. Dentro de la OMS, el aborto inseguro es un procedimiento para interrumpir un embarazo no deseado, practicado por personas sin capacitación o experiencia y/o en condiciones que carecen de estándares médicos mínimos. ${ }^{1}$
Día con día un buen número de mujeres se enfrentan a un embarazo no deseado, lo cual les lleva a la decisión de practicarse un aborto, método que muchas veces se realiza en las peores circunstancias. Sabemos que para un sector de la sociedad el aborto conlleva una condena moral; esta situación conduce a las mujeres a poner en gran riesgo su salud recurriendo a la automedicación que les puede provocar el aborto y otras complicaciones o también las arrastra a asistir a lugares insalubres donde les realizan el aborto en condiciones clandestinas y por lo tanto peligrosas que incrementan la morbilidad y mortalidad materna.

El aborto ha sido el último recurso para muchas mujeres que quieren evitar un dolor mayor, como lo plantean algunos estudios en el tema embarazo no deseado, 
que se define como el rechazo activo y razonado de la concepción durante las primeras semanas de gestación.

De acuerdo a International Projects Assistance Services (IPAS) en México, el aborto y sus complicaciones son la $5^{a}$ causa de muerte materna, responsable del $7.2 \%$ de las defunciones, el $14 \%$ de estas muertes sucedieron en el Distrito Federal, que es la entidad con mayor porcentaje de estos casos, el $45 \%$ se da en mujeres de 20 a 29 años, la mayor parte de la defunciones (64\%) en mujeres sin derechohabiencia a la seguridad social. ${ }^{2}$

La OMS conceptualiza el aborto como la expulsión de un embrión o un feto que pese $500 \mathrm{~g}$ o menos (percentil 50 de una gestación de 20 semanas). Por ILE se entiende como la interrupción de un embarazo que está permitida por la ley. En el caso de las menores de edad se requiere el acompañamiento de sus padres o tutores para realizar la interrupción legal del embarazo. ${ }^{3}$

En 1869 la iglesia católica romana prohibió el aborto y lo evaluó a sanción universal. En Inglaterra y EUA era legal o tolerado ampliamente el aborto; sin embargo, como parte de la restructuración general de las leyes sobre delitos, en Inglaterra se promulgó un estatuto que calificó de ilegales a todos los abortos. En 1821 el estado de Connecticut de EUA, promulgó la primera ley sobre abortos; tal práctica se volvió ilegal en todo el país, excepto para salvar la vida de la madre, hasta que la Suprema Corte de EUA decidió, en 1973, adoptar medidas definitivas; en muchos Estados legalmente sólo podían realizarse abortos con fin terapéutico. Hasta esa fecha la definición legal más común de ese tipo de aborto era la terminación del embarazo antes de la viabilidad fetal, para salvar la vida de la madre. ${ }^{4}$

La Constitución Política de los Estados Unidos Mexicanos, en el artículo $4^{\circ}$, establece como garantías individuales el derecho de toda persona a la protección de la salud y a decidir de manera libre, responsable e informada sobre el número y el espaciamiento de sus hijos, ${ }^{5}$ $y$, es responsabilidad del Gobierno del Distrito Federal, a través de la Secretaría de Salud del Distrito Federal, garantizar su ejercicio para lograr el bienestar físico, mental y social de la mujer y contribuir así al pleno ejercicio de sus capacidades.

Los argumentos expuestos en la Suprema Corte de Justicia de la Nación demostraron que las reformas efectuadas el 24 de abril de 2007 por la Asamblea Legislativa del Distrito Federal, fueron realizadas dentro del marco de las facultades de las diputadas y diputados locales para legislar en materia de salud pública, y que dichas reformas no sólo sí son acordes a la Constitución Política de los Estados Unidos Mexicanos, sino que resultan idóneas para el ejercicio

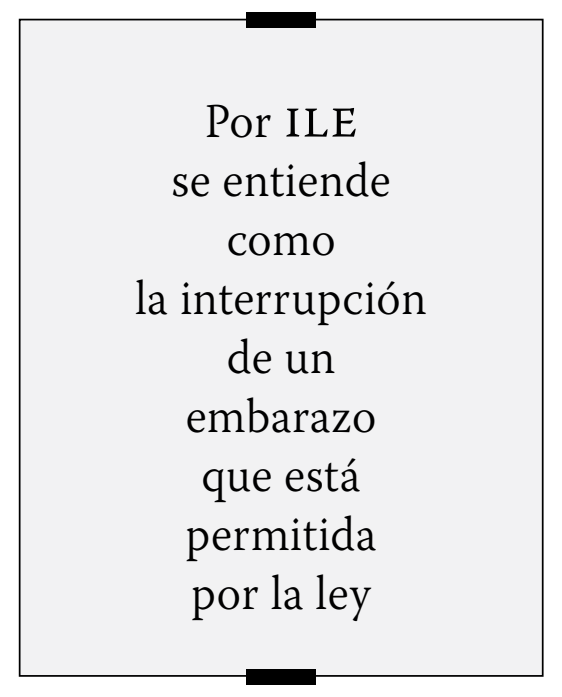

de los derechos de las mujeres a una maternidad libre, informada y voluntaria, legalizando el programa ILE. ${ }^{6}$

La ILE se permite en las entidades federativas en los siguientes casos:

En toda la República Mexicana, en caso de violación puede interrumpir el embarazo.

En la mayoría de los Estados se permite la ILE, cuando la vida de la mujer corre grave peligro si no se interrumpe el embarazo.

La despenalización de la interrupción del embarazo antes de las 12 semanas de gestación (SDG) en la Ciudad de México reportada en el número 75 en mayo de 2007 en la Gaceta Oficial del Distrito Federal, ${ }^{7}$ representa un paso más en la lucha que han dado las mujeres del movimiento feminista por el respeto de su cuerpo y al derecho a elegir voluntaria y libremente la maternidad o a decidir la interrupción de un embarazo, fundamentada en una serie de derechos humanos ya reconocidos: el derecho a la igualdad y la no discriminación, a la vida, a no ser sometida a torturas, a la libertad y seguridad personales, a no ser objeto de injerencias arbitrarias en la vida y la familia, a la libertad de pensamiento, conciencia y religión, derechos que forman parte de la Declaración Universal de los Derechos Humanos y del Pacto Internacional de Derechos Civiles y Políticos.

En el D.F, las mujeres tienen la opción de interrumpir su embarazo hasta las $12 \mathrm{SDG}$, sin que sea considerado un delito. Aunque al solicitar el servicio de ILE las mujeres no tienen que especificar un motivo, entre las razones más frecuentes por las que una mujer pue- 
de optar por interrumpir su embarazo están:

1. No contar con los recursos para mantener a una criatura en ese momento,

2. Que el embarazo afecte sus planes de estudio,

3. Que haya fallado el método anticonceptivo

4. Que haya tenido relaciones sexuales sin protección,

5. Porque ya tiene los hijos que desea o,

6. Simplemente porque no quiere tener hijos.

La ILE se realiza en todos los hospitales de la Secretaría de Salud del DF de forma gratuita. La cifra sobre la interrupción legal del embarazo en el D. F. desde su legalización el 24 de abril de 2007 al 24 de agosto de 2009 es de 29,212 casos reportados. ${ }^{8}$

La decisión de interrumpir un embarazo no es fácil, ésta se debe tomar en libertad, de a cuerdo con la conciencia, sin ningún tipo de presión por parte de familiares, amistades o cualquier otra persona. Para poder decidir de manera

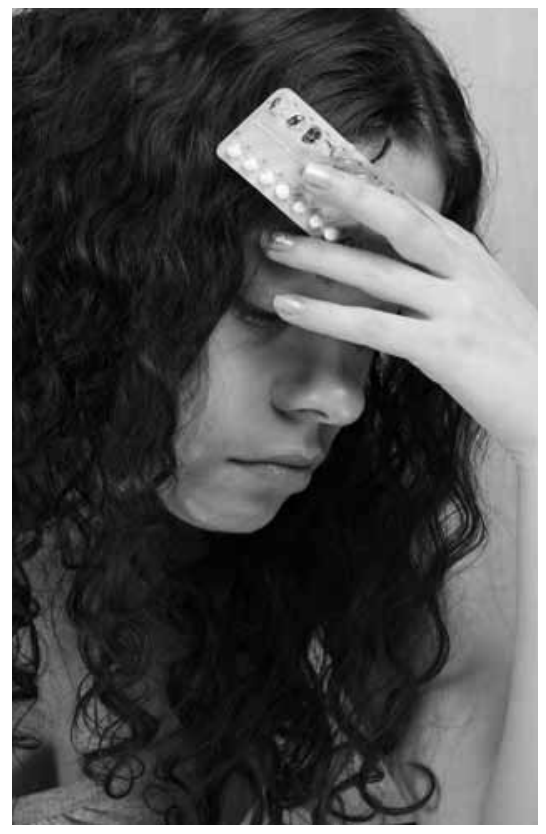

libre y responsable se requiere de información clara, veraz, objetiva, precisa y oportuna sobre el procedimiento médico para la ILE, sus riesgos y consecuencias.

\section{ANTECEDENTES}

\section{DE LA LITERATURA}

A partir de los años cincuenta cuando los países de Europa central comenzaron a admitir el aborto por voluntad de las mujeres- se inició la liberalización de la leyes de aborto, sea a través de la despenalización o la legalización. En los años sesentas y setentas el resto de los países industrializados revisaron sus leyes y admitieron, o bien más causales que permiten el aborto, o bien liberaron por completo su legislación respectiva. Asimismo, ciertos países en desarrollo como China e India hicieron lo propio. Desde 1994, año en que tuvo lugar la Conferencia Internacional sobre la Población y el Desarrollo en El Cairo, 12 países han liberalizado sus leyes, mientras que sólo cuatro hicieron cambios restrictivos en materia de aborto. ${ }^{9}$

En la actualidad tenemos que casi dos terceras partes de las mujeres del mundo residen en países donde el aborto puede obtenerse a solicitud o por una amplia gama de razones socioeconómicas o personales. ${ }^{10}$

Los países que cuentan con servicios de aborto legal y seguro tienen, por lo general, menos complicaciones y muertes relacionadas con los embarazos, así como niveles menores de infanticidio y abandono de infantes. En estos países, los abortos son realizados por personal médico capacitado, de manera que la intervención es segura, está disponible y resulta menos costosa.
Con todo esto, se ha mejorado la salud de las mujeres y de sus hijos. ${ }^{11}$

El objetivo de la despenalización del aborto en el Distrito Federal fue por la necesidad de disminuir la alta incidencia de morbi-mortalidad producidos por el aborto inseguro. En 2005, la mortalidad materna fue de 63.3 muertes por 100,000 nacidos vivos; en el Distrito Federal fue de 57.6, mayor a la de otros estados. Entre 1990 y 2005; en el D.F., el aborto fue la tercera causa de muerte materna, mientras que a nivel nacional constituyó la quinta causa de muerte. ${ }^{12}$

La tasa de aborto inducido en México se incrementó en un 64 por ciento en los últimos 16 años, al pasar de 25 a 33 abortos por cada mil mujeres, tasa superior a la de países en desarrollo y a la latinoamericana (de 29 y 31 por cada mil respectivamente. ${ }^{13}$

El estudio "Estimaciones del aborto inducido en México: ¿qué tanto ha cambiado entre $1990 \mathrm{y}$ 2006?", realizado por las investigadoras de El Colegio de México, Fátima Juárez, del Instituto Alan Guttmacher y del Population Council, sentó presedentes en este ámbito. Se enfatizó que en el mismo periodo (1990 a 2006) también se incrementó anualmente el número de mujeres que recurrían al aborto inducido. De 533 mil en 1990, pasó a 875 mil en 2006.

En el mismo periodo, reporta el estudio, casi 150 mil mujeres recibieron tratamiento en hospitales públicos en el país, como consecuencia de abortos realizados en condiciones clandestinas. Además, sólo una de cada seis mujeres que tuvieron un aborto inducido en el 2006 fue tratada en un hospital público por complicaciones. ${ }^{14}$ 
En una encuesta reciente de IPsos- Bimsa para Population Council, una de las más completas sobre el tema, se ponderan causales para determinar el apoyo o no a un aborto, la muestra estuvo integrada por hombres y mujeres encontrando que 6 de cada 10 sujetos apoyan que el aborto se realice bajo ciertas circunstancias. $\mathrm{Y}$ al menos 5 de cada 10 sujetos justifica que las causales para acudir al aborto sean la falla del método anticonceptivo, la decisión propia de la mujer, falta de recursos económicos y si fuese madre soltera. ${ }^{15}$

En el D. F. el procedimiento de ILE se realizaba exclusivamente en Hospitales del Gobierno local, pero para aumentar la accesibilidad del procedimiento y evitar la saturación de los servicios hospitalarios, se reestructuró el Módulo Quirúrgico del Centro de Salud T-III "Beatriz Velasco de Alemán" para constituir una Clínica de Atención Integral de la Salud Reproductiva. Quienes durante mayo 2008 y febrero 2009 reportan que de las mujeres que asistieron a realizarse ILE mantiene preeminencia las jóvenes, ya que en el grupo de menores de 30 años se concentra el $78 \%$ de las atenciones, observando mayor porcentaje (34.4\%) las mujeres de 21 a 25 años. El $54.8 \%$ se reportan como solteras y 39.7 casadas o en unión libre. Con respecto al lugar de residencia, hay un predominio natural de pacientes del Distrito Federal y del Estado de México (juntos concentran el 93.9\%), y de las foráneas la mayor parte provienen de Puebla, Jalisco, Morelos y Michoacán. En el caso de las pacientes a las que no se realizó procedimiento, el mayor porcentaje fue por exceder las 12 semanas de gestación. ${ }^{16}$

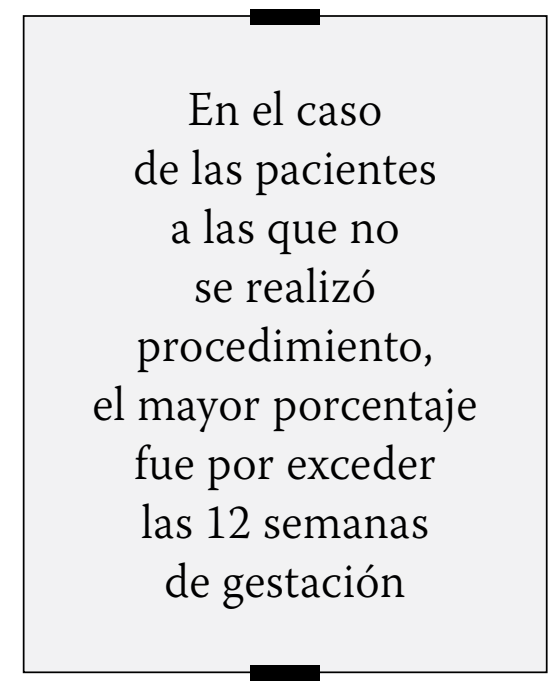

La problemática de la legalización del aborto ha tenido una larga y sustanciosa historia que ha culminado en leyes que protegen a la mujer en el derecho de decidir sobre su cuerpo y cuantos hijos desea tener. Por lo que el presente estudio tuvo como fin hacer una característica de las mujeres que hacen uso del programa de Interrupción legal del Embarazo aplicado en el D.F.

\section{METODOLOGÍA}

Estudio cuantitativo, descriptivo y transversal, realizado en un hospital Materno Infantil del Sector Salud del gobierno del Distrito Federal, durante el primer trimestre de 2009. Muestreo por conveniencia encuestando a 61 mujeres embarazadas con menos de 12 semanas de gestación, que deseaban ingresar al programa ILE y aceptaron participar en el estudio, previo consentimiento informado. Cabe señalar que por motivos de inconsistencia o con datos incompletos se tuvieron que eliminar 5 cuestionarios quedando con una población total de 56 mujeres. Se realizó y aplicó un instrumento con 27 ítems, el cual coincide con datos considerados en la hoja de registro de las mujeres que ingresan al programa de ILE; el cual fue sometido a jueceo de 5 expertos. Tras las observaciones y su evaluación el instrumento fue reestructurado y aplicado para el levantamiento de datos.

Posterior a la aplicación su confiabilidad alcanzó un alpha de Cronbach de 0.749 , lo que da cuenta de la consistencia interna de los ítems.

El procesamiento estadístico de los datos fue a través del programa SPSS versión 12.0 por medio de recursos descriptivos tales como tablas, medidas de resumen, y medidas de dispersión.

\section{RESULTADOS}

Las mujeres que acudieron al servicio de consulta externa para ingresar al programa ILE durante el 1er trimestre de 2009 oscilaron en un rango de edad entre 16 y 42 años, teniendo mayor frecuencia aquellas que están entre los 19 y 24 años $(26$ mujeres $=46.4 \%)$. El $46 \%$ de las encuestadas se dedican a labores del hogar y $25 \%$ eran estudiantes. El nivel escolar con el que contaban las mujeres que ingresaron al programa ILE era para el 36\% de preparatoria terminada, $23 \%$ secundaria terminada y $16 \%$ licenciatura. El estado civil que tenían las mujeres del estudio fue en el $50 \%$ de los casos solteras y el otro $50 \%$ correspondió a mujeres unión libre o casadas.

Respecto a la vida sexual activa, se tiene que la edad de inicio de la vida sexual activa osciló desde los 13 y hasta los 28 años, teniendo que el mayor porcentaje correspondió a las mujeres que iniciaron su vida sexual a los 18 años con un $32 \%$, a los 17 y 16 años $16 \%$ respectivamente y a los 15 años, $14 \%$ de las encuestadas. El $48 \%$ ha tenido 
1 pareja sexual, el $26 \%$ con 2 parejas sexuales, aunque hay quienes han tenido 3, 4 ó hasta 5 parejas sexuales.

Con respecto a la convivencia se encontró que el $31 \%$ vivía con su pareja o esposo, otro $30 \%$ vivía con los padres, el resto vivía sola, con hijos u otros familiares.

Respecto a su perfil reproductivo se detectó que el 32\% de las mujeres sometidas a ILE durante los tres primeros meses del año aun no tenían hijos vivos, $26 \%$ tenían 2 hijos, $17 \%$ tenían uno y cinco hijos respectivamente, el $7 \%$ tenia 3 hijos e incluso un $1 \%$ había tenido ya, 7 hijos. Cincuenta mujeres refirieron no haber tenido abortos, 4 más aceptan haber tenido un aborto y dos mujeres admiten haber tenido 2 y 4 abortos respectivamente. Con respecto a las semanas de gestación con que contaban, 33 de ellas se encontraban entre 7 y 9 SDG, aunque había quien contaba con 4 y hasta 11 SDG reportadas por ultrasonido y confirmadas por clínica.
El lugar de procedencia variaba de diversas delegaciones del D. F. y municipios del Estado de México. Al indagar acerca de métodos anticonceptivos empleados para evitar el embarazo se señalaron los comercialmente usados desde parches, inyecciones, preservativo, pastillas y DIU, sin embargo el $23 \%$ reportó no emplear ningún método para control de la natalidad. Aunque las mujeres conocen de la existencia del programa ILE y los resultados de éste, el 68\% refiere no conocer los recursos a emplear en el programa.

La forma en la que se enteraron de la existencia de la ILE principalmente fue a través de diversas fuentes como medios de comunicación, amistades e incluso familiares. El programa ILE incluye como parte fundamental la Consejería, a razón de ello, 46 de las entrevistadas reportan que la información proporcionada fue suficientemente clara, sin embargo las 10 mujeres restantes aseguraron que la información no fue clara. Se cuestionó a las mujeres que en el caso de no existir el Programa ILE acudirían a otro lado, el $66 \%$ refirió que si, si habrían buscado otra alternativa para interrumpir el embarazo, siendo principalmente la asistencia a médico particular; el $34 \%$ restante reportó que no acudiría a interrumpir su embarazo de no existir la ILE.

Dentro de las principales razones que las mujeres que se incorporaron al estudio reportaron como motivo para acudir al programa de interrupción legal del embarazo se encuentran: problemas económi$\cos (36 \%)$, embarazo no deseado (20\%), paridad satisfecha (20\%), problemas de salud (14\%), temor a ser madre soltera (5\%) y temor por la edad y sus efectos al bebé (5\%) (ver gráfica No. 1). El principal sentimiento que despierta el ingreso al programa ILE es tranquilidad (82\%) a estar consciente y segura de lo que se esta realizando. Así mismo el 70\% de ellas consideran que las mujeres que son sometidas al programa no requieren ayuda psicológica tras haber realizado interrup-

\section{GRAFICO No. 1.}

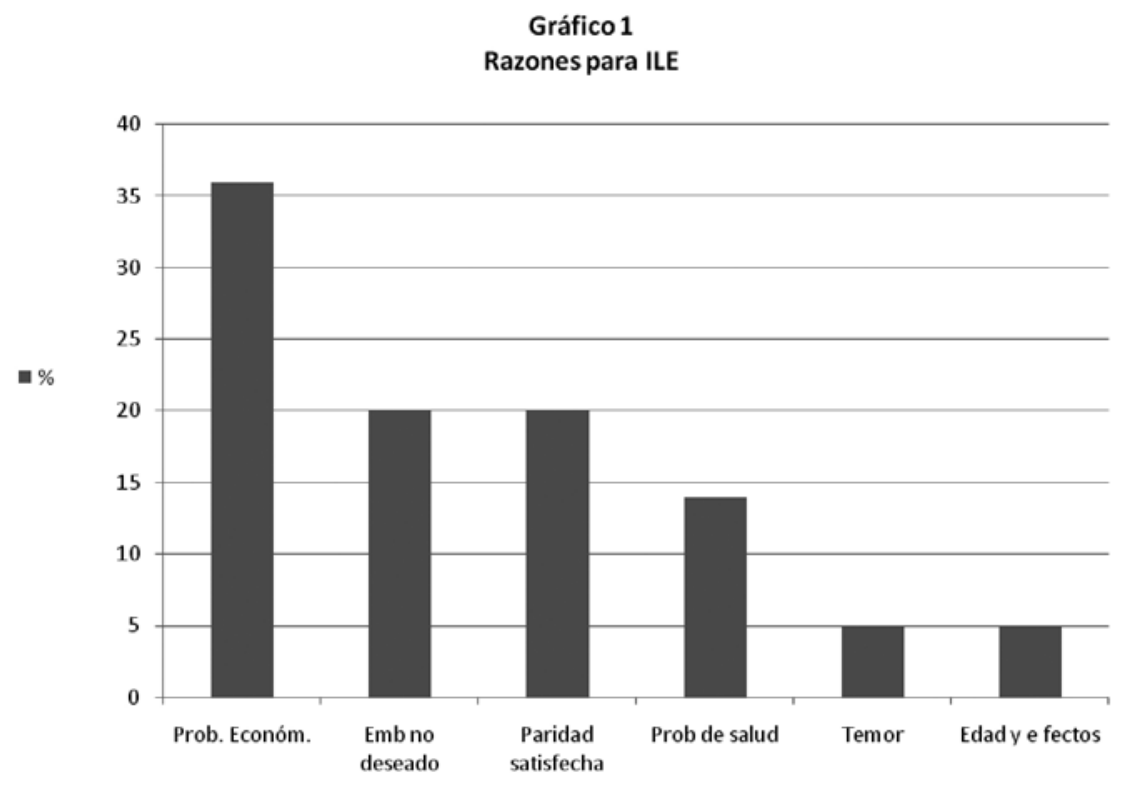


ción legal del embarazo, aunque el $30 \%$ asegura si necesitarlo. El 100\% de las mujeres sometidas a ILE aseguran haber sido tratadas con ética y dignidad por parte del personal que le atiende.

\section{DISCUSIÓN}

De los datos obtenidos con respecto a Interrupción Legal del Embarazo, podemos notar que los resultados en cuanto a la edad, condición civil, lógicamente semanas de gestación y nivel de estudios es similar lo reportado por la Jurisdicción Sanitaria de la Delegación Venustiano Carranza con lo encontrado en el presente estudio. Respecto a las razones que llevan a la mujer a tomar la desición de ingresar al programa ILE tales como embarazo no deseado y los problemas económicos, ello corresponde con los resultados de la encuesta IPSOS- Bimsa para Population Council en la población Mexicana. ${ }^{17}$

Se debe de tomar en cuenta que de acuerdo con los datos de Gutmacher ${ }^{18}$, donde se muestra un incremento del $62 \%$ de abortos inducidos en mujeres mexicanas en un periodo de 16 años, ante cifra $\tan$ alarmante donde la mujer puede encontrarse en grave riesgo, es correspondiente con la incidencia de solicitudes de ingreso al programa en hospitales del Gobierno Mexicano. En donde al menos en los resultados del presente estudio se acumuló una muestra de 56 mujeres que acudieron a un solo hospital del D.F. en un periodo de 3 meses.

Esto nos habla de que la solicitud del servicio se mantiene en una población homogénea en la que se debe asegurar calidad en la atención y fortalecer las medidas de prevención.

Acerca de la consejería y apoyo psicológico que mostró que 7 de cada 10 mujeres opinaron no requerirla, se debe aclarar, que se encuentra en los lineamientos generales de organización y operación de los servicios de salud para la interrupción legal del embarazo en el Distrito Federal, en su artículo $3^{\circ}$, romano $I^{1,9}$ que la mujer debe recibir consejería para proporcionarle orientación, asesoría e información objetiva, veraz, suficiente y oportuna sobre los procedimientos, riesgos, consecuencias y efectos; situación que es llevada a cabo en las mujeres que solicitan el servicio, pero que además debiera de ser un servicio posterior a la intervención para evitar desviaciones en el uso de este programa.

\section{CONCLUSIONES}

La interrupción legal del embarazo es un programa del gobierno del Distrito Federal que ha apoyado la disminución de la morbi-mortalidad de mujeres en edad reproductiva, se realiza con discreción, confidencialidad, privacidad, respeto, equidad, objetividad, neutralidad y libertad. La mujer debe de cubrir requisitos de firmar el consentimiento informado, haber recibido consejería, tener dictamen médico de embarazo con semanas de gestación hasta la segunda semana.

Las usuarias que acuden por este servicio en el D.F. corresponden con las razones expuestas en estudios previos, la demanda es alta y se tiene en cuenta los beneficios de la despenalización del aborto sobre las disminución de las muertes maternas.

El mejoramiento de la calidad de atención a la salud reproductiva debe ser creciente, se debe priorizar en la prevención de embarazos no deseados, orientando y educando a la población y ofreciendo métodos de planificación a hombres y mujeres con vida sexual activa.

Las mujeres que tienen embarazos no deseados deben tener la seguridad de contar con acceso a la información fidedigna y consejería eficiente y un trato humano que incluye respeto a la dignidad y sentido ético.

En ningún caso se debe promover el aborto como método de planificación familiar, más allá de esto se deben fortalecer acciones que contribuyan a la toma de conciencia de las implicaciones y responsabilidades que involucra la vida sexual activa.

Con la infraestructura adecuada y procedimientos bien diseñados e implementados por personal calificado, se puede ofrecer seguridad en la realización del procedimiento, por ello es necesario mantener los esfuerzos para contar con los servicios necesarios, al mismo tiempo que es conveniente mantener la capacitación permanente del personal que asiste a las mujeres que asisten para ILE.

\section{REFERENCIAS Bibliográficas}

1 Ipas México. Protegiendo la salud de las mujeres. Promoviendo el respeto a los derechos reproductivos. La enfermera en la Salud reproductiva: sus intervenciones en la atención obstétrica y síndrome de aborto. 2009

2 Ipas México. Protegiendo la salud de las mujeres. Promoviendo el respeto a los derechos reproductivos. La enfermera en la Salud reproductiva: sus intervenciones en la 
atención obstétrica y síndrome de aborto. 2009

3 Bulletin of the World Healt Organization, 2000, 78 (5), pags. 569, 580,581 ,

4. F. Gary Cunningham, 2006 "Obstetricia de Wlliams: Aborto" 22a Edición, MacGraw Hill Interamericana Pá: 241.

5 Gaceta Oficial del Distrito Federal. Consultado el 7 de ferero de 2009. Disponible en: www.shottama. org/.../Pdfs/GACETA\%20OFICIAL\%20DEL\%20DISTRITO\%20 FED

6 Gaceta Oficial del Distrito Federal. Consultado el 7 de ferero de 2009. Disponible en: www.shottama. org/.../Pdfs/GACETA\%20OFICIAL\%20DEL\%20DISTRITO\%20 FED

7 Instituto de las Mujeres del D.F. Acerca de la Despenalización de la Interrupción del Embarazo. Consultado el 18 de marzo de 2009 en: http://www.inmujer.df.gob.mx/ int_embarazo/acerca_de.html.

8 Ahued, A. A. 2009. Presentación de resultados en el Foro: Dos años de ejercer nuestros derechos: Interrupción Legal del Embarazo en el D. F. México. GIRE. [on line] Consultado el 29 de agosto de 2009 en http://www.gire.org.mx/publica2/ ILE_DF_Cifras_200409.pdf

9 Katzive, Laura, "Abortion Law Changes since 1994", Center for Reproductive Rights, documento sin publicar, 2003.
10 Abortion in Law, History \& Religion, Childbirth by Choice Trust, Toronto, 1995

11 Anika Rahman, Laura Katzive y Stanley K. Henshaw, "A Global Review of Laws on Induced Abortion, 1985- 1997", en International Family Planning Perspectives, vol. 24, núm. 2, Nueva York, The Alan Guttmacher Institute, junio, 1998, pp. 56-64.

12 Godinez L. L. (2006). CIMACNOTICIAS. MÉXICO Por aumento de aborto inducido, urge homologar ILE en el país. [on line] Consultado el 10 de marzo de 2010 de World Wide Web de: http://www.alterinfos.org/spip.php?article2797

13 Colegio Mexicano de Anestesiología. (2008) .Boletín. La interrupción legal del embarazo. Época II Vol III Núm 2 - Abril-Mayo. [on line] Consultado el 10 de marzo de 2010 de World Wide Web de: http://www.comexan.com.mx/boletin.htm

14Grupo de Información en Reproducción Elegida A.C.(2008) .Boletín de prensa El número de abortos inducidos en México aumentó un 64 por ciento. [on line] Consultado el 10 de marzo de 2010 de World Wide Web de: http://www.alterinfos.org/spip.php?article2797

15 Instituto de las Mujeres de la Ciudad de México. (2006). Cifras en torno a la despenalización del aborto [on line] Consultado el 10 de marzo de 2010 de
World Wide Web de: http://www. inmujer.df.gob.mx/int_embarazo/ documentos/aumento_causales. html

16 Secretaría de Salud del Distrito Federal. Jurisdicción Sanitaria Venustiano Carranza. Interrupción Legal del Embarazo (ILE) Construyendo el acceso a una Atención Primaria de Salud con Calidad y Seguridad para el Paciente. Centro de Salud T-III "Beatriz Velasco de Alemán” México, D.F. 2009

17 Instituto de las Mujeres de la Ciudad de México. (2006). Cifras en torno a la despenalización del aborto [on line] Consultado el 10 de marzo de 2010 de World Wide

Web de: http://www.inmujer. df.gob.mx/int_embarazo/documentos/aumento_causales.html 18 Grupo de Información en Reproducción Elegida A.C.(2008) .Boletín de prensa El número de abortos inducidos en México aumentó un 64 por ciento. [on line] Consultado el 10 de marzo de 2010 de World Wide Web de: http://www.alterinfos.org/spip.php?article2797

19 Colegio Mexicano de Anestesiología. (2008) .Boletín. La interrupción legal del embarazo. Época II, Vol III Núm 2 - Abril-Mayo. [on line] Consultado el 10 de marzo de 2010 de World Wide Web de: http://www.comexan.com.mx/boletin.htm 